REVISTA CIENCIAS BIOMÉ DICAS

PRESENTACIÓN DE CASOS CLÍNICOS

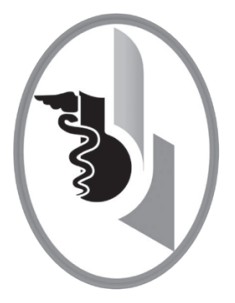

\title{
EPENDIMOMA MIXOPAPILAR OSTEOLÍTICO GIGANTE DEL SACRO: UNA FORMA AGRESIVA DE UN TUMOR BENIGNO
}

\author{
GIANT OSTEOLYTIC MYXOPAPILLARY EPENDYMOMA OF THE \\ SACRUM: AN AGGRESSIVE FORM OF A BENIGN TUMOR
}

Tuñón-Pitalúa Martha Cecilia ${ }^{1}$

Ruiz-Cáez Karina María²

Niño-Hernández Lucía Mercedes²

Piña-Cabrales Sandra ${ }^{3}$

Alcalá-Cerra Gabriel ${ }^{4}$

Remolina-López Anderson Julián ${ }^{4}$

Correspondencia: mtunon@gmail.com

Recibido para evaluación: marzo - 25 - 2014. Aceptado para publicación: octubre - 15 - 2015.

\section{RESUMEN}

Introducción: el ependimoma mixopapilar (EM) es un tumor glial ependimario que afecta predominantemente a los adultos jóvenes, se origina en el filum terminale, la cauda equina y el cono medular.

Caso clínico: se presenta el caso de una paciente en quien durante el estudio de un dolor radicular se logra detectar ependimoma mixopapilar gigante, el cual comprometía gran parte del hueso sacro, mostrando un comportamiento localmente agresivo con intensa osteólisis.

Conclusión: se discutieron las posibles teorías que explican la presencia de estos tumores a nivel sacro, en especial la presencia de remanentes embrionarios de tejido ependimario en los niveles más caudales del canal vertebral y los posibles mecanismos que explican el comportamiento agresivo de estos tumores histológicamente benignos. Rev.cienc.biomed. 2015; 6(2):348-353

\section{PALABRAS CLAVE}

Columna vertebral; Ependimoma mixopapilar; Neoplasia extradural; Sacro; Región sacrococcígea.

\section{SUMMARY}

Introduction: myxopapillary ependymoma (MP) is a glial ependymal tumor that mainly affects young adults. The tumor originates in the filum terminale, the cauda equina and the conus medullaris.

\footnotetext{
Médico. Especialista en Patología. Neuropediatra. Facultad de Medicina. Universidad de Cartagena. Cartagena. Colombia.

Médico. Estudiante de Postgrado. Patología. Universidad de Cartagena. Cartagena. Colombia.

Médico. Servicio de Neurocirugía. Fundación Centro Colombiano de Epilepsia y Enfermedades Neurológicas - FIRE. Cartagena de Indias. Colombia.

4 Médico. Estudiante de postgrado. Neurocirugía. Facultad de Medicina. Universidad de Cartagena. Colombia.
} 
Case record: the case presented in a patient in whom a giant myxopapillary ependymoma was detected during a medical checkup for root pain. The tumor involved the sacral bones showing a locally aggressive behavior with intense osteolysis.

Conclusion: possible theories that explain the presence of these tumors in sacral bones were discussed, focusing in the presence of embryonic remnants of ependymal tissue in the most caudal levels of the spinal canal, and the possible mechanisms that explain the aggressive behavior of these histologically benign tumors. Rev.cienc.biomed.2015; 6(2):348-353

\section{KEYWORDS}

Spine; Myxopapillary

Sacrococcygeal region.

ependymoma; Epidural neoplasm; Sacrum;

\section{INTRODUCCIÓN}

El ependimoma mixopapilar (EM) es una lesión tumoral infrecuente de linaje glial que afecta predominantemente a los adultos jóvenes, incluida actualmente en el grupo de los tumores ependimarios de la clasificación internacional de la Organización Mundial de la Salud (OMS). Clínicamente se caracteriza por crecimiento lento y localización casi exclusiva en la médula espinal terminal o cono medular, cola de caballo y el filum terminale. La serie más extensa fue desarrollada por el grupo cooperativo de la Rare Cancer Network durante 38 años consecutivos donde se encontraron 85 casos, de los cuales el 53\% eran de localización lumbosacra, $41 \%$ toracolumbar y $6 \%$ cérvico-torácico ( 1 ).

En ocasiones, los EM pueden extenderse hacia los huesos sacros, siendo difícil determinar si el tumor tiene origen intra o extradural. Generalmente se encuentran confinados al canal espinal y la destrucción de las estructuras óseas, vertebrales o ilíacas adyacentes al tumor, son eventos extremadamente raros (2).

\section{CASO CLÍNICO}

Paciente femenino de 53 años de edad, con cuadro clínico de seis años de evolución consistente en dolor lumbar irradiado a miembro inferior derecho, asociado a claudicación neurogénica e incontinencia urinaria.

En el examen físico se encontró paresia (fuerza muscular 4/5) e hipotrofia de los músculos cuádriceps, tibial anterior, sural y gastronemio derechos. Fue encontrada hi-

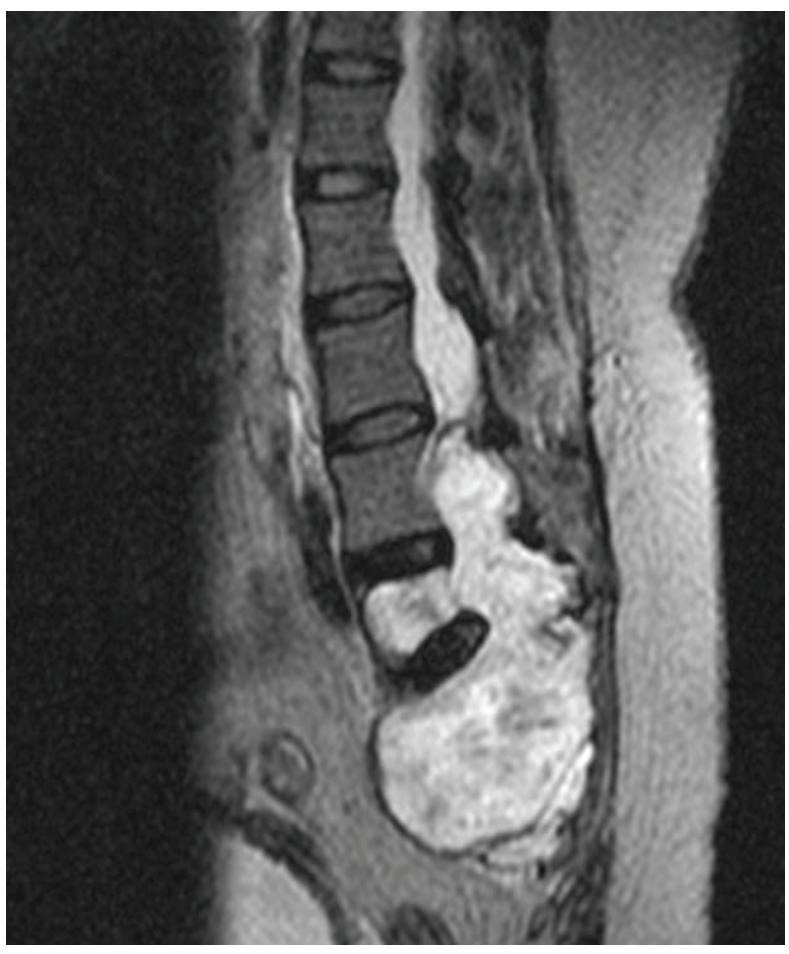

Figura No 1. Imagen de un corte sagital por resonancia magnética. Lesión tumoral lítica comprometiendo los cuerpos vertebrales L5 y S1. Ocupación casi completa del canal vertebral.

poestesia para todas las modalidades en los dermatomas correspondientes a L4, L5, S1, S2 y S3. Se encontró además, hiporeflexia patelar derecha y arreflexia aquílea bilateral.

Fue realizada una tomografía computarizada abdominal que demostró lesión osteolítica en columna lumbosacra que se extiende desde L4 hasta S1 comprometiendo canal raquídeo. Las imágenes de resonancia magnética nuclear de columna lumbosacra con contraste demostraron lesión tumoral lítica comprometiendo los cuerpos vertebrales L5 
y S1. El tumor ocupaba casi completamente el canal vertebral (Figura No 1).

Por las características imagenológicas y la extensión de la lesión se realizó laminectomía S1 y resección parcial, por no ser posible identificar claramente las raíces nerviosas para su preservación. Posterior al procedimiento, la paciente no presentó déficit neurológico adicional.

Se examinaron muestras del tejido. Microscópicamente se observó lesión tumoral glial constituida por células cúbicas con núcleos ovoides formando papilas con centros vasculares hialinizados en la mayor parte de las áreas y en otras con centros mucoides. En algunos focos se observaron estructuras rosetoides.

La marcación inmunohistoquímica reveló positividad intensa y difusa de las células tumorales para la proteína glial fibrilar acídica, positividad focal para EMA, Ki-67 menor de $1 \%$, sin marcación a la sinaptofisina. Los hallazgos morfológicos fueron consistentes con EM (Figura No 2).
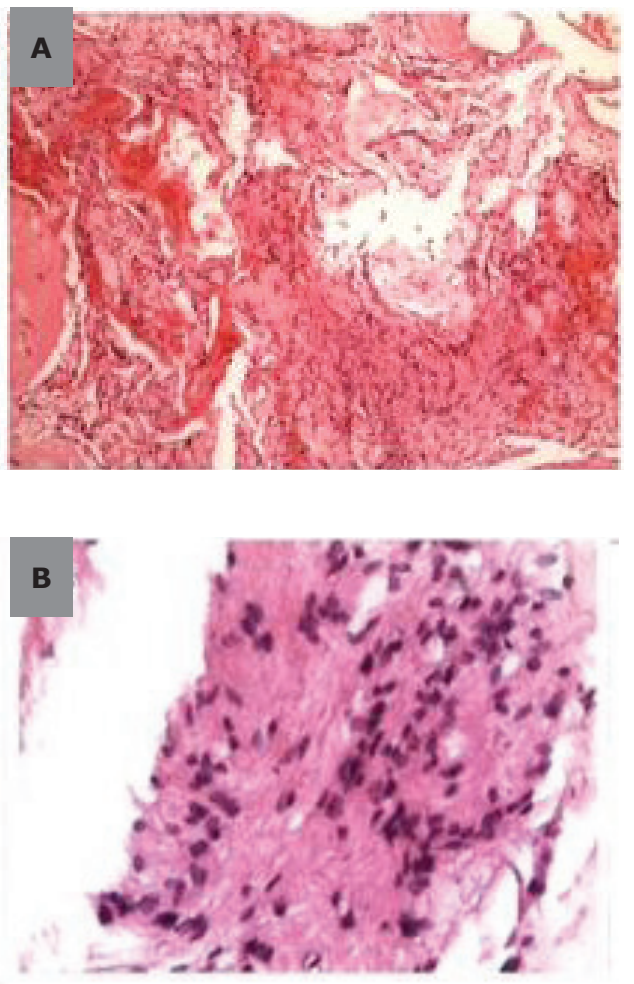
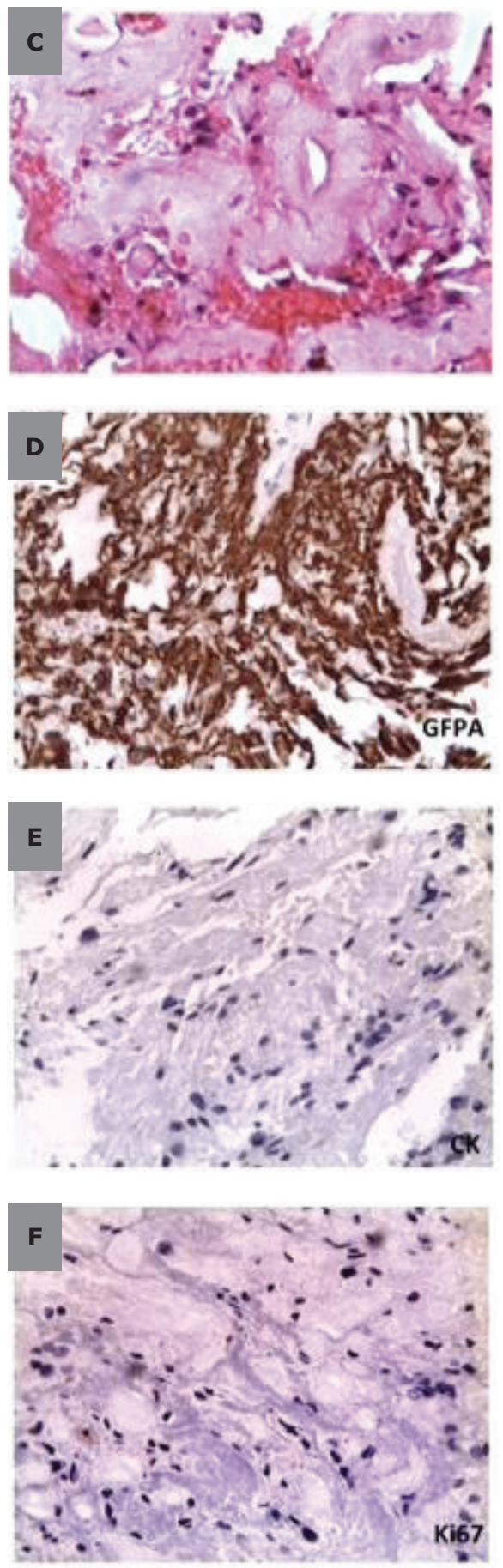

Figura No 2. Ependimoma mixopapilar. (A) Lesión compuesta por células tumorales dispuestas en patrón de rosetas rodeando los vasos hialinizados y áreas mixoides con hemorragia, tinción $\mathrm{HE} \times 5 \mathrm{X}$; (B) Células tumorales elongadas con cromatina fina, con distribución perivascular, tinción HE $\times 40 X$; (C) Prominente hialinización vascular, tinción HE $\times 40 X$; (D) Inmunoreactividad fuerte y difusa de las células tumorales con la proteína glial fibrilar ácida, anticuerpos GFPA $\times 40$; (E) Ausencia de reactividad a la citoqueratina, anticuerpos citoqueratina $\times 40$; (F) Baja inmunoreactividad con el Ki-67, anticuerpos $\mathrm{Ki}-67 \times 40$. 
ISSN: 2215-7840, 6(2), julio-diciembre 2015, Tuñón-Pitalúa Martha Cecilia, Ruiz-Cáez Karina María, Niño-Hernández Lucía Mercedes, Piña-Cabrales Sandra, Alcalá-Cerra Gabriel, Remolina-López Anderson Julián

\section{DISCUSIÓN}

La identificación de un EM por fuera del neuroeje central puede ser debida a una extensión metastásica de un tumor intradural, aunque de forma menos frecuente la totalidad del tumor puede originarse por fuera de la duramadre (3). Gupta (3), Quraishi (2) y sus respectivos colaboradores refrendaron (2), que la diferencia entre un tumor puramente extradural y una extensión de un tumor intradural es muy difícil de establecer. Para lo cual sugieren que en los casos en donde el mayor volumen tumoral se encuentra en el sacro, -desde donde se extiende intraduralmente- su origen debe ser atribuido a células ubicadas al interior de las vértebras sacras (3).

La localización primaria extradural estaría relacionada con derivados embrionarios de células ependimarias, probablemente, restos extradurales del filum terminale o el vestigio coccígeo medular que sufrirían el proceso de transformación neoplásica (2). El tejido ependimario que puede encontrarse en la cauda equina, y particularmente en el filum terminale, es un remanente embrionario de tejido neural. Durante el tercer mes del desarrollo embrionario el extremo distal de la médula espinal se encuentra en el punto más caudal del canal vertebral.

El crecimiento desproporcionado de la columna vertebral en comparación con la médula espinal explica la presencia de la ubicación del cono medular a nivel del punto medio del canal espinal lumbar durante la vida fetal avanzada. Por este motivo, algunas células ependimarias pueden quedar rezagadas, fijándose entre el cono medular y la segunda vértebra sacra $(4,5)$.

Los EM extradurales en la zona sacra son muy raros. Los sitios frecuentes de localización son: 1) el canal espinal extradural en asociación con la parte dural del filum terminal; 2) huesos sacros; 3) cavidad pélvica anterior hasta el sacro y 4) tejidos subcutáneos detrás de la columna vertebral y huesos sacros (6).

Aunque los EM pueden diseminarse al resto de la médula espinal, la metástasis por fuera del neuroeje es muy rara. Los principales órganos diana de las metástasis son el parénquima pulmonar, hígado, huesos y ganglios linfáticos, lo cual sugiere una vía de diseminación predominantemente hematógena (2).

También existen reportes de casos localizados en el cuarto ventrículo $(7,8)$, bulbo raquídeo (9), ovarios, hoz cerebral (10) y mediastino (11), posiblemente originadas en heterotopias ependimarias como resultado del cierre incompleto del arco neural $(2,6)$.

Histológicamente el EM está compuesto por células tumorales con hallazgos consistentes con lesión benigna y tasa de proliferación muy baja, sin embargo, en algunos casos su comportamiento clínico y biológico es agresivo con alta tasa de recidiva e invasión a las estructuras óseas vecinas (12).

Al examen macroscópico el EM se caracteriza por ser masa lobulada o fusada, ricamente vascularizada, a menudo encapsulada, de consistencia blanda, con áreas de hemorragia y degeneración mucinosa. Histopatológicamente, se caracteriza por células cuboidales o alargadas, de núcleo ovoide, con cromatina finamente granular; dispuestas radialmente alrededor de tallos de estroma mixoide ricamente vascularizado, quienes configuran las estructuras papilares.

Adicionalmente, se encuentran acúmulos de material mixoide, el cual tiñe con azul alcian y ácido peryódico de Schiff, formando espacios micro-quísticos $(13,14)$. Algunas lesiones pueden mostrar escasas papilas, incluso pueden estar ausentes; casos en los cuales son remplazadas por fascículos de células alargadas o por matriz extracelular con escaso componente glial (12). Frecuentemente se observa trombosis, hemorragias y depósitos de hemosiderina (14). El pleomorfismo nuclear es leve, con mínima actividad proliferativa y escasa mitosis.

Los estudios de inmunohistoquímica revelan positividad para proteína glial fibrilar acídica, S-100, vimentina, y negatividad para citoqueratina, lo cual permite diferenciarlo histológicamente de los cordomas, condrosarcomas mixoides y meningiomas cordoides 
(15). Sin embargo, ocasionalmente puede presentar inmunopositividad para citoqueratinas [CAM 5.2, AE1 AE3, CK7] y antígeno de membrana epitelial [EMA], diagnosticán- dose de forma errónea como un carcinoma metastásico. No obstante, el perfil de coexpresión de GFAP y S100 permite su diferenciación. Tabla No 1 (16).

\begin{tabular}{|l|l|l|l|}
\hline \multicolumn{5}{|c|}{ TABLA NO $\mathbf{1}}$. \\
PERFIL INMUNOHISTOQUÍmICO PARA DIFERENCIACIÓN DEL EPENDIMOMA \\
MIXOPAPILAR
\end{tabular}

Solo existen algunos reportes de casos de EM que producen cambios osteolíticos en las vértebras adyacentes, el sacro y/o los huesos ilíacos $(2-5,13,14)$. La serie más numerosa fue reportada por Quraishi y colaboradores, quienes lograron identificar tan solo seis casos. Se cree que el comportamiento destructivo de las estructuras óseas circundantes estaría relacionado con el volumen tumoral, ya que usualmente estas lesiones han alcanzado un volumen suficiente para ocupar todo el diámetro del canal vertebral (2).

El tratamiento ideal de los EM es la resección completa, lo cual se relaciona con bajo riesgo de recidivas y largos periodos libres de enfermedad, pero se realiza en menos de la mitad de los casos que se presentan y por ello, requiere tratamiento complementario con radioterapia. Los tumores que presentan osteólisis tienen una alta tasa de recidiva y metástasis sistémica, debido a que su resección completa es técnicamente difícil (3).

\section{CONCLUSIÓN}

El ependimoma mixopapilar (EM) es un tumor glial ependimario que afecta predominantemente a los adultos jóvenes. El diagnóstico histopatológico se puede realizar mediante microscopía de luz, si se identifica la disposición celular típica de las células ependimarias formando papilas en un fondo mixoide. No obstante, en algunos casos es imprescindible realizar inmunohistoquímica para el diagnóstico diferencial con cordomas, condrosarcomas mixoides, meningiomas cordoides y carcinomas metastásicos.

CONFLICTO DE INTERESES: ninguno que declarar.

FINANCIACIÓN: recursos propios de los autores. Estudios de laboratorio, insumos hospitalarios, medicamentos y honorarios profesionales aportados dentro de la atención asistencial.

\section{REFERENCIAS BIBLIOGRÁFICAS}

1. Pica A, Miller R, Villa S, Kadish SP, Anacak $Y$, Abusaris $H$, et al. The results of surgery, with or without radiotherapy, for primary spinal myxopapillary ependymoma: a retrospective study from the rare cancer network. Int J Radiat Oncol Biol Phys. 2009;74:1114-20.

2. Quraishi NA, Wolinsky JP, Bydon A, Witham T, Gokaslan ZL. Giant destructive myxopapillary ependymomas of the sacrum. J Neurosurg Spine. 2010;12:154-9.

3. Gupta R, Rishi A, Suri V, Sharma MC, Gupta A, Garg A, et al. Sacral myxopapillary ependymoma with extensive osteolysis. J Neurooncol. 2008;86:349-52.

4. Chung JY, Lee SK, Yang KH, Song MK. Subcutaneous sacrococcygeal myxopapillary ependymoma. AJNR Am J Neuroradiol. 1999;20:344-6.

5. Biagini R, Demitri S, Orsini U, Bibiloni J, Briccoli A, Bertoni F. Osteolytic extra-axial sacral myxopapillary ependymoma. Skeletal Radiol. 1999;28:584-9.

6. Fassett DR, Schmidt MH. Lumbosacral ependymomas: a review of the management of intradural and extradural tumors. Neurosurg Focus. 2003;15:E13.

7. Chakraborti S, Govindan A, Alapatt JP, Radhakrishnan M, Santosh V. Primary myxopapillary ependymoma of the fourth ventricle with cartilaginous metaplasia: a case report and review of the literature. Brain Tumor Pathol. 2011. 
ISSN: 2215-7840, 6(2), julio-diciembre 2015, Tuñon-Pitalúa Martha Cecilia, Ruiz-Cáez Karina María, Niño-Hernández Lucía Mercedes, Piña-Cabrales Sandra, Alcalá-Cerra Gabriel, Remolina-López Anderson Julián

8. Lim SC, Jang SJ. Myxopapillary ependymoma of the fourth ventricle. Clin Neurol Neurosurg. 2006;108:211-4.

9. DiLuna ML, Levy GH, Sood S, Duncan CC. Primary myxopapillary ependymoma of the medulla: case report. Neurosurgery. 2010;66:E1208-9; discussion E9.

10. Tseng YC, Hsu HL, Jung SM, Chen CJ. Primary intracranial myxopapillary ependymomas: report of two cases and review of the literature. Acta Radiol. 2004;45:344-7.

11. Estrozi B, Queiroga E, Bacchi CE, Faria Soares de Almeida V, Lucas de Carvalho J, Lageman GM, et al. Myxopapillary ependymoma of the posterior mediastinum. Ann Diagn Pathol. 2006;10:283-7.

12. Schittenhelm J, Becker R, Capper D, Meyermann R, Iglesias-Rozas JR, Kaminsky J, et al. The clinico-surgico-pathological spectrum of myxopapillary ependymomas--report of four unusal cases and review of the literature. Clin Neuropathol. 2008;27:21-8.

13. Shors SM, Jones TA, Jhaveri MD, Huckman MS. Best cases from the AFIP: myxopapillary ependymoma of the sacrum. Radiographics. 2006;26:111-6.

14. Sonneland PR, Scheithauer BW, Onofrio BM. Myxopapillary ependymoma. A clinicopathologic and immunocytochemical study of 77 cases. Cancer. 1985;56:883-93.

15. Cho HY, Lee M, Takei H, Dancer J, Ro JY, Zhai QJ. Immunohistochemical comparison of chordoma with chondrosarcoma, myxopapillary ependymoma, and chordoid meningioma. Appl Immunohistochem Mol Morphol. 2009;17:131-8.

16. Hussein SA, Sur M. Cytokeratin positivity in myxopapillary ependymoma--a potential diagnostic pitfall. Diagn Pathol. 2008;3:40.

Es el órgano de información científica de la Facultad de Medicina de la Universidad de Cartagena. Colombia.

Publique su trabajo en esta revista enviando su manuscrito a: revistacienciasbiomedicas@unicartagena.edu.co www.revistacienciasbiomedicas.com www.revistacienciasbiomedicas.com.co

Revista ciencias Biomédicas es una publicación independiente, imparcial, abierta, revisada por pares, de elevada visibilidad internacional, con circulación online e impresa. Publica artículos en todas las modalidades universalment aceptadas en inglés y en español, de temas referentes con todas las ciencias biomédicas, incluyendo ámbitos clínicos, epidemiológicos o de estudios básicos.

El sistema de gestión de manuscritos es rápido y justo.

Revista Ciencias Biomédicas está incluida en varias bases de datos latinoamericanas e internacionales.

Antes de enviar su manuscrito, revise las recomendaciones para los autores, presentes en www.revistacienciasbiomedicas.com www.revistacienciasbiomedicas.com.co 